\title{
Mercury revealed
}

The smallest planet is yielding some big surprises. One year after NASA's MESSENGER probe entered orbit around Mercury, planetary scientists have been stunned by evidence of recent geological activity and an interior far different from any other planet.

Mercury has long been the enigmatic oddball of the inner Solar System. In the 1970s, the Mariner 10 spacecraft flew past Mercury three times, gathering data that indicated the planet was denser than any other and that an iron-rich core made up a large fraction of its interior. Impact craters scarring its surface told of billions of years of meteorite bombardment.

Now, MESSENGER is bringing Mercury into far sharper focus. By shooting lasers from the probe's near-polar orbit and measuring how long it takes the pulses to bounce back, mission scientists have compiled the first detailed topographic model of the planet's northern hemisphere (Science Express 336, 217-220; 2012).

The map reveals that the elevation of Mercury's surface varies by less than $9.9 \mathrm{~km}$ across the hemisphere, making Mercury's northern half smoother than the Moon or Mars by a factor of 2 and 3, respectively. In one spot, the northern lowlands display a broad region, around $950 \mathrm{~km}$ across, that is

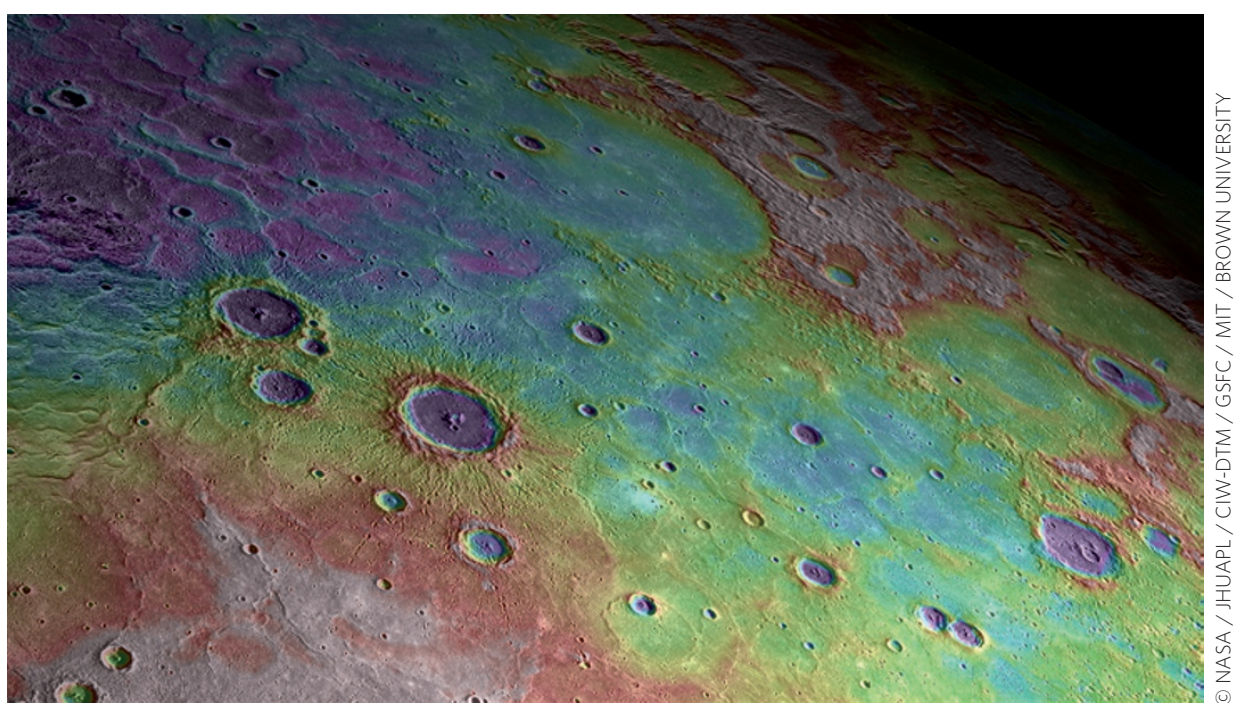

about $1.5 \mathrm{~km}$ higher than its surroundings. Elsewhere, the interior of the 1,500-km-wide Caloris basin - Mercury's largest - has been uplifted to the point that some of the basin's floor is higher than the rim.

Both the rise on the northern plains and the changes at Caloris must have taken place after the period of heavy meteorite bombardment, in the early days of the Solar System, and after the vast volcanic plains

\section{The journalist's take}

Little, faraway and often hidden in the Sun's glare, Mercury isn't an easy planet to love. And until MESSENGER, the few images that existed of the planet were let's face it - boring. Mercury looked grey and dead on its surface, with only big craters providing some visual relief.

But now high-resolution pictures are flowing back from NASA's probe, and reporters are starting to perk up. The pair of recent Science papers, along with other Mercury studies announced the same week at the Lunar and Planetary Science Conference in Texas, garnered headlines worldwide. In part, that's because the new results show that Mercury is more active, dynamic and tantalizing than scientists had thought. Reporters find it hard to resist that sort of storyline.

Furthermore, planetary science has always had more than its fair share of media attention compared with other areas of geoscience. Spacecraft journeys provide a compelling narrative, from launch to arrival to discovery, around which journalists can frame conveniently packaged stories. Missions also carry a hefty dose of technological wonder, where humanity tests the bounds of its robotic capabilities in otherworldly environments. The probe ceases to be a machine and instead takes on the hopes and dreams of the public, a fact that the media can exploit to produce popular stories.

Finally, perhaps more than any other research agency, NASA has mastered the art of public relations. The new Mercury findings were released along with webcast press conferences and slickly packaged multimedia extras. For harried editors looking for a feel-good story with good art, Mercury could not be beaten.

Thanks to this, the smallest planet may be coming into the media limelight after all. had formed. This relative youth of the topographic features indicates that Mercury was geologically active later in its history than scientists had thought.

Similarly, Mercury's interior structure has turned out to be more complicated than once believed. The MESSENGER team used radio tracking of the spacecraft to calculate how small changes in its orbit translated into variations in the planet's gravitational pull. The resulting gravity field model shows several large anomalies in the northern hemisphere, including ones associated with the northern rise and Caloris basin (Science Express 336, 214-217; 2012).

Calculations of the planet's spin indicate that, to yield the observed gravity, it must have an extremely large core occupying about 85 per cent of the planetary radius. The solid outer shell must also be quite dense. But because the surface rocks don't contain much iron to account for that density, the MESSENGER team suggests there could be a layer of iron sulphide surrounding the large core.

This leaves the problem of how much mantle Mercury actually has. If most of the planet is core topped by iron sulphide, there is relatively little mantle to be displaced and uplifted, as seen on the surface at places like Caloris.

Answers may yet come. MESSENGER's mission has been extended for another year, so it can keep gathering clues to the nature of this oddball little planet.

Alexandra Witze covers the Earth and other sciences for the US magazine Science News. 